\title{
EQUIVARIANT DIFFEOMORPHISMS WITH SIMPLE RECURRENCES ON TWO-MANIFOLDS
}

\author{
BY
}

W. DE MELO, G. L. DOS REIS AND P. MENDES ${ }^{1}$

\begin{abstract}
We consider the class of diffeomorphisms, on compact two-dimensional manifolds, which are invariant under the action of a compact Lie group $G$ and whose nonwandering set consists of a finite number of $G$-orbits. We describe the modulus of stability of almost all diffeomorphisms in this class.
\end{abstract}

Introduction and statement of results. Let $G$ be a compact Lie group acting smoothly on a smooth closed connected two-dimensional manifold $M$. We say that a $G$-equivariant diffeomorphism of $M$ has simple recurrences if its nonwandering set consists of a finite number of normally hyperbolic critical elements. Denote by $\mathscr{A}$ the set of $G$-equivariant diffeomorphisms with simple recurrences whose stable and unstable manifolds of its critical elements are $G$-transversal. There are many diffeomorphisms in $\mathscr{A}$ which are not structurally stable. However, for some of them it may be possible to parametrize all the topological conjugacy classes in their neighborhood by finitely many real parameters. In this case we say that they have finite modulus of stability. Otherwise, their modulus of stability is infinite.

In this paper we study the modulus of stability of diffeomorphisms in a dense set $\mathscr{B} \subset \mathscr{A}$.

Now we will state our result in a precise way. A $C^{\infty}$-diffeomorphism $f: M \rightarrow M$ is $G$-equivariant if $f(g \cdot x)=g \cdot f(x)$ for all $x \in M$ and $g \in G$. The set of such diffeomorphisms with the $C^{\infty}$ topology is denoted by $\operatorname{Diff}_{G}(M)$. An element $f$ in Diff $_{G}(M)$ induces a $G \times \mathbf{Z}$-action on $M$ given by $(g, n) \cdot x=g \cdot f^{n}(x)$. A critical element of $f$ is a compact $G \times \mathbf{Z}$-orbit. A critical element $\sigma$ of $f$ is normally hyperbolic if there is a continuous invariant splitting of the tangent bundle of $M$ over $\sigma, T_{\sigma} M=T \sigma \oplus E^{s} \oplus E^{u}$, and constants $c>0,0<\lambda<1$, such that $\left\|D f_{x}^{n} v^{s}\right\| \leq$ $c \lambda^{n}\left\|v^{s}\right\|, v^{s} \in E^{s}$, and $\left\|D f_{x}^{-n} v^{u}\right\| \leq c \lambda^{n}\left\|v^{u}\right\|, v^{u} \in E^{u}$, for all $x \in \sigma$ and all $n>0$. The stable manifold of $\sigma, W^{s}(\sigma)=\left\{y \in M\right.$ : distance of $f^{n}(y)$ to $\sigma$ tends to zero when $n \rightarrow+\infty\}$, has $G, f$-invariant foliation $\mathscr{F}_{\sigma}^{s s}(f)$ whose leaves are the strong stable manifolds of the points in $\sigma$. Similarly, the unstable manifolds of $\sigma$ have a $G$, $f$-invariant foliation $\mathscr{F}_{\sigma}{ }^{u u}(f)$. Here, in dimension two, we have that $\sigma$ is either a periodic orbit or a finite union of circles. In the first case $\sigma$ is just a hyperbolic orbit and $\mathscr{F}_{\sigma}^{s s}(f)\left(\mathscr{F}_{\sigma}{ }^{u u}(f)\right)$ is a finite number of submanifolds. In the second case $\sigma$ is an

Received by the editors April 13, 1984 and, in revised form, August 21, 1984.

1980 Mathematics Subject Classification. Primary 58F10; Secondary 57S15.

Key words and phrases. Compact Lie groups, equivariant diffeomorphisms, structural stability, modulus of stability.

${ }^{1}$ The second and third authors were partially supported by CNPq (Brazil). 
attractor (or a repellor) and $\mathscr{F}_{\sigma}^{s s}(f)\left(\mathscr{F}_{\sigma}^{u u}(f)\right)$ is a foliation with one-dimensional leaves. These leaves are just stable (unstable) manifolds of the points in $\sigma$.

For the notion of $G$-transversality between stable and unstable manifolds of normally hyperbolic critical elements, see Bierstone [B] and Field [F1, F2]. For two-dimensional manifolds that notion is equivalent to the one called "stratumwise transversality". This property is defined as follows. If $H=G_{x}$ is the isotropy subgroup of a point $x \in M$, then the conjugacy class $(H)$ of $H$ is called the type of the $G$-orbit $G(x)$. The union $M_{(H)}$ of all $G$-orbits of type $(H)$ is a differentiable $G$-fibre bundle with the $G$-orbits as fibres. The manifold $M$ is stratified by orbit type. If $W \subset M$ and $H$ is a subgroup of $G$, we set $W_{(H)}=W \cap M_{(H)}$. Let $W^{u}$ and $W^{s}$ be the unstable and stable manifolds of a normally hyperbolic critical element of $f \in \operatorname{Diff}_{G}(M)$. We say that $W^{u}$ is stratumwise transverse to $W^{s}$ if $W_{(H)}^{u}$ is transverse to $W_{(H)}^{s}$ in $M_{(H)}$, and write $W^{u} \cap{ }_{G}^{\mathrm{T}} W^{s}$. We notice that the stratification of $M$ by the orbit type is finite and there is a stratum which is open and dense. It consists of $G$-orbits of maximal dimension called principal $G$-orbits (see [ $\mathbf{B r}]$ ).

For $f \in \mathscr{A}$, let $\left\{\sigma_{1}, \ldots, \sigma_{n}\right\}$ be the set of critical elements of $f$. We say that $\sigma_{i} \leq \sigma_{j}$ if $W^{u}\left(\sigma_{i}\right)$ has a $G$-transverse intersection with $W^{s}\left(\sigma_{j}\right)$. We put $\sigma_{i}<\sigma_{j}$ if $\sigma_{i} \leq \sigma_{j}$ and $\sigma_{i} \neq \sigma_{j}$. If a critical element of $f$ is a periodic orbit of saddle type we say that it is a saddle. An $r$-chain of saddles of $f \in \mathscr{A}$ is a collection of saddles $\mathcal{O}\left(p_{1}\right)<\cdots<$ $\mathcal{O}\left(p_{r}\right)$, such that there is no saddle $\mathcal{O}(p)$ with $\mathcal{O}\left(p_{i}\right)<\mathcal{O}(p)<\mathcal{O}\left(p_{i+1}\right), i=1, \ldots, r$ -1 , and $\mathcal{O}\left(p_{r}\right) \nless \mathcal{O}\left(p_{1}\right)$. Here, $p \in M$ is a saddle point and $\mathcal{O}(p)$ is its orbit. If $\mathcal{O}\left(p_{1}\right)=\mathcal{O}\left(p_{r}\right)$, we have an $(r-1)$-cycle. Let $p_{i}=f\left(p_{i}\right), i=1,2$, be saddles of $f \in \mathscr{A}$. If $W^{u}\left(p_{1}\right)-\left\{p_{1}\right\}$ and $W^{s}\left(p_{2}\right)-\left\{p_{2}\right\}$ have a common connected component $\gamma_{12}$ we say that $\left(p_{1}, p_{2}, \gamma_{12}\right)$ (or $\left.\left(p_{1}, p_{2}\right)\right)$ is a saddle connection of $f$. By the $G$-transversality property $\gamma_{12}$ is contained in a one-dimensional stratum $M_{(H)}$. In a similar way we can define saddle connection for periodic orbits.

Let $p$ be a hyperbolic fixed point of a $C^{2}$-diffeomorphism $f$. By a theorem of Hartman [Ha], there is a $C^{1}$-diffeomorphism $\varphi$ on a neighborhood of $p$ conjugating $f$ to its linear part. If $W^{u}(p)$ and $W^{s}(p)$ are imbedded submanifolds of $M$ and do not accumulate in each other, we may extend $\varphi$ to a $C^{1}$-diffeomorphism $\varphi$ of a neighborhood $U$ of $W^{u}(p) \cup W^{s}(p)$ onto an open set $V$ of $\mathbf{R}^{2}$ conjugating $f$ and $D f(p)$. The Euclidean metric of $\mathbf{R}^{2}$ induces via $\varphi$ a metric $d_{p}$ on $U$ which we call linearizing metric at $p$. Notice that the linearizing metric is not unique, but if $d_{p}^{\prime}$ is another linearizing metric at $p$, there exist positive real numbers $\lambda^{s}, \lambda^{u}$ such that $d_{p}^{\prime}\left(x^{s}, y^{s}\right)=\lambda^{s} d_{p}\left(x^{s}, y^{s}\right)$ if $x^{s}, y^{s} \in W^{s}(p)$ and $d_{p}^{\prime}\left(x^{u}, y^{u}\right)=\lambda^{u} d_{p}\left(x^{u}, y^{u}\right)$ if $x^{u}$, $y^{u} \in W^{u}(p)$. Let $\left(p_{1}, p_{2}, \gamma_{12}\right)$ be a saddle connection of a $C^{2}$-diffeomorphism $f$ of $M$. Let $\beta_{f}: \gamma_{12} \rightarrow \mathbf{R}$ be the function defined by

$$
\beta_{f}(q)=\lim _{\substack{x \rightarrow q \\ x \notin \gamma_{12}}} \frac{d_{p_{2}}(x, q)}{d_{p_{1}}(x, q)},
$$

where $d_{p_{i}}$ is a linearizing metric at $p_{i}, i=1,2$. This function is well defined up to multiplication by positive constant. In [dM-D] it is proved that if $f$ is $C^{r+1}, r \geq 1$, then $\beta_{f}$ is in fact $C^{r}$. Hence, for each $q \in \gamma_{12}$ the function

$$
T_{f, q}(x)=\left[\beta_{f}(x) / \beta_{f}(q)\right]^{1 / \log \left|\lambda_{1}\right|}
$$


is $C^{r}$ and does not depend on the choice of the metrics. Here, $\lambda_{1}$ is the contracting eigenvalue of $D f_{p_{1}}$. This is the transition function defined in [dM].

Now we define the subsets $\mathscr{C} \subset \mathscr{B}$ of $\mathscr{A}$, which are the main objects in our result.

An element $f \in \mathscr{A}$ is in $\mathscr{B}$ when the transition functions associated to saddle connections of $f$ are Morse functions.

An element $f \in \mathscr{B}$ is in $\mathscr{C}$ when

(1) the chains of saddles of $f$ have at most one saddle connection;

(2) the chains of saddles of $f$ with one saddle connection have length less than 5 ;

(3) if $\left(p_{1}, p_{2}, p_{3}, p_{4}\right)$ is a 4-chain of $f$ with one saddle connection, then $\left(p_{2}, p_{3}\right)$ is its saddle connection;

(4) if $f$ has a 4-chain with one saddle connection, then the associated transition function has no critical points.

We have the following

THEOREM. Suppose the principal orbits of $G$ have dimension zero. Then a diffeomorphism $f$ in $\mathscr{B}$ has finite modulus of stability if and only if $f \in \mathscr{C}$.

REMARK. The fact that the $G$-transversality property fails to be open in $\operatorname{Diff}_{G}(M)$, even for elements in $\mathscr{B}$, is contained in the proof of our Theorem.

The proof of the Theorem consists of a globalization of several "semilocal" cases studied in $\$ 2$. In that section we examine the contribution of saddle connections to moduli of stability and prove the Theorem. In $\$ 1$ we state some known facts about moduli of stability of diffeomorphisms on two-dimensional manifolds, useful for our purpose, mostly proved in $[\mathbf{d M}] . \S 3$ is devoted to the case where the principal $G$-orbits have dimension greater than zero.

As a general reference for classical notions on dynamical systems we refer the reader to [P-dM and F2] for $G$-equivariant dynamical systems.

We are grateful to J. Palis for helpful discussions.

1. Preliminaries. In this section $f, \bar{f} \in \mathscr{A}$ have saddle connections $\left(p_{1}, p_{2}, \gamma_{12}\right)$ and $\left(\bar{p}_{1}, \bar{p}_{2}, \bar{\gamma}_{12}\right)$, and $\lambda_{i}, \mu_{i},\left|\lambda_{i}\right|<1,\left|\mu_{i}\right|>1$, and $\bar{\lambda}_{i}, \bar{\mu}_{i},\left|\bar{\lambda}_{i}\right|<1,\left|\bar{\mu}_{i}\right|>1$, are the eigenvalues of $D f\left(p_{i}\right)$ and $D \bar{f}\left(\bar{p}_{i}\right)$ respectively, $i=1,2$.

(1.1) A necessary condition for the existence of a conjugacy $h$ between $f$ and $\bar{f}$ (a homeomorphism $h$ such that $h f=\bar{f} h$ ) from a neighborhood of $c l\left(\gamma_{12}\right)$ onto a neighborhood of $\operatorname{cl}\left(\bar{\gamma}_{12}\right)$ is that $\log \left|\bar{\mu}_{2}\right| / \log \left|\bar{\lambda}_{1}\right|=\log \left|\mu_{2}\right| / \log \left|\lambda_{1}\right|$, called the eigenvalue condition $[\mathbf{P}]$.
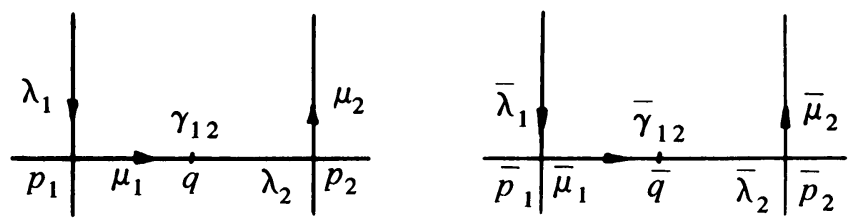

Figure 1 
The following facts are proved in $[\mathbf{d M}]$.

(1.2) If (i) $\left(\log \left|\bar{\mu}_{2}\right| / \log \left|\bar{\lambda}_{1}\right|\right)=\left(\log \left|\mu_{2}\right| / \log \left|\lambda_{1}\right|\right)$, and (ii) there is a homeomorphism $\Psi: \gamma_{12} \rightarrow \bar{\gamma}_{12}$ such that $\Psi(q)=\bar{q}$ and $T_{f, q}=T_{\bar{f}, \bar{q}} \circ \Psi$, then there is a conjugacy between $f$ and $\bar{f}$ in neighborhoods of $\operatorname{cl}\left(\gamma_{12}\right)$ and $\operatorname{cl}\left(\bar{\gamma}_{12}\right)$.

(1.3) If $\log \left|\mu_{2}\right| / \log \left|\lambda_{1}\right|$ is irrational and there exists a conjugacy between $f$ and $\bar{f}$ sending $q$ to $\bar{q}$, then there exists a homeomorphism $\Psi: \gamma_{12} \rightarrow \bar{\gamma}_{12}$ such that $T_{f, q}=$ $T_{\bar{f} \cdot \bar{q}} \circ \Psi$ and $\Psi(q)=\bar{q}$.

REMARK. Let $\left(p_{1}, p_{2}, \gamma_{12}\right)$ be a saddle connection of diffeomorphism $f \in \mathscr{A}$. Suppose that for some $q \in \gamma_{12}, T_{f, q}$ is a Morse-function and has a critical point (in fact $T_{f, q}$ must have at least two $f$-orbits of critical points). Then it follows from (1.2) and (1.3) that the modulus of stability of $f$ in a neighborhood of $\gamma_{12}$ is equal to the number of $f$-orbits of critical points of $T_{f, q}$. In fact, let $\bar{f}$ be a diffeomorphism near $f$ having a saddle connection $\left(\bar{p}_{1}, \bar{p}_{2}, \bar{\gamma}_{12}\right)$. By varying $\bar{q}$ in $\bar{\gamma}_{12}$ all critical values of $T_{\bar{f}, \bar{q}}$ change in the same direction. Therefore we can choose $\bar{q} \in \bar{\gamma}_{12}$ such that one of the critical values of $T_{\hat{f}, \bar{q}}$ is equal to the corresponding critical value of $T_{f, q}$. Hence the conjugacy invariants are $\log \left|\mu_{2}\right| / \log \left|\lambda_{1}\right|$ and the remaining critical values of $T_{f, q}$ in the fundamental domain $[q, f(q)] \subset \gamma_{12}$.

(1.4) Let $\log \left|\mu_{2}\right| / \log \left|\lambda_{1}\right|$ be irrational and $h$ be a conjugacy between $f$ and $\bar{f}$ such that

$$
h\left(p_{i}\right)=\bar{p}_{i}, \quad i=1,2, \quad \text { and } \quad h(q)=\bar{q} \quad \text { for } q \in \gamma_{12} .
$$

Then $d_{\bar{p}_{1}}\left(h(x), \bar{p}_{1}\right) /\left[d_{p_{1}}\left(x, p_{1}\right)\right]^{c}$ is constant on each connected component of $W^{s}\left(p_{1}\right)-\left\{p_{1}\right\}$ and $d_{\bar{p}_{2}}\left(h(x), \bar{p}_{2}\right) /\left[d_{p_{2}}\left(x, p_{2}\right)\right]^{c}$ is constant on each connected component of $W^{u}\left(p_{2}\right)-\left\{p_{2}\right\}$. Here $c=\left(\log \left|\bar{\mu}_{2}\right| / \log \left|\mu_{2}\right|\right)=\left(\log \left|\bar{\lambda}_{1}\right| / \log \left|\lambda_{1}\right|\right)$ and $d_{p_{i}}, d_{\bar{p}_{i}}$ are linearizing metrics at $p_{i}, \bar{p}_{i}, i=1,2$.

(1.5) If $W^{s}\left(p_{1}\right)$ (resp. $W^{u}\left(p_{2}\right)$ ) contains a denumerable set of orbits belonging to unstable (resp. stable) manifolds of saddles of $f$, then the modulus of stability of $f$ is infinity.

(1.6) Let $\beta_{f}: \gamma_{12} \rightarrow \mathbf{R}$ be the function defined by

$$
\beta_{f}(q)=\lim _{\substack{x \rightarrow q \\ x \notin \gamma_{12}}} \frac{d_{p_{2}}(x, q)}{d_{p_{1}}(x, q)},
$$

where $d_{p_{i}}, i=1,2$, are linearizing metrics. We have $\beta_{f}(f(q))=\left|\mu_{2}\right| \beta_{f}(q) /\left|\lambda_{1}\right|$. In particular, $\lim _{q \rightarrow p_{1}} \beta_{f}(q)=0$ and $\lim _{q \rightarrow p_{2}} \beta_{f}(q)=+\infty$. Notice that if $x_{n} \notin \gamma_{12}$ is such that $d_{p_{1}}\left(x_{n}, \gamma_{1,2}\right) \rightarrow 0$ and $\left(d_{p_{2}}\left(x_{n}, \gamma_{12}\right) / d_{p_{1}}\left(x_{n}, \gamma_{12}\right)\right) \rightarrow a$, then the limit points of $\left\{x_{n}\right\}$ are contained in $\beta_{f}^{-1}(a)$. In particular, if $\beta_{f}$ is monotone, then $x_{n}$ converges.

Now we recall some properties of invariant foliations. Proofs can be found in the appendix of [dM-vS].

(1.7) An unstable foliation $\mathscr{F}^{u}$ at a saddle point $p$ of $f$ is an invariant foliation of a neighborhood of $W^{s}(p)$ such that each leaf intersects $W^{s}(p)$ transversally at a unique point. In dimension two, if $f$ is $C^{2}$, the leaves of $\mathscr{F}^{u}$ are $C^{2}$ and the line field tangent to the leaves is $C^{1}$ in the complement of $W^{u}(p)$, then it is $C^{1}$. This fact allows us to prove Hartman's $C^{1}$-linearization theorem using stable and unstable foliations. In particular, given an unstable foliation with the above properties there is 
a linearizing metric $d_{p}$ such that the function $x \rightarrow d_{p}\left(x, W^{u}(p)\right)$ is constant on the leaves of $\mathscr{F}^{u}$.

Now a final word about the local structure of the $G$-action when $G$ is finite. For $x \in M$ let $H=G_{x}$ be its isotropy group. By the "slice theorem" [ $\mathrm{Br}$ ] there is a neighborhood of $x$ where $G_{x}$ acts orthogonally.

(1.8) If $x$ is in a 1-dimensional stratum, then $G_{x}$ acts as a reflection about $M_{(H)}$. So in Figure 1 the behavior of $f$ in the lower side of $\gamma_{12}$ is a reflection of its behavior in the upper side.

2. Proof of the Theorem. We start studying the moduli of stability of diffeomorphisms in $\mathscr{B}$ in neighborhoods of saddle connections.

The following lemma is a direct consequence of (1.5).

LEMMA 2.1. Let $f \in \mathscr{B}$ and assume that $\left(p_{1}, p_{2}, p_{3}, p_{4}\right)$ is a 4-chain of saddles of $f$ which presents $\left(p_{1}, p_{2}\right)$ or $\left(p_{3}, p_{4}\right)$ as its unique saddle connection. Then the modulus of stability of $f$ is infinite.

Let $f \in \mathscr{B}$ and let $p_{i}$ be a saddle of $f$. We denote by $D_{ \pm}^{u}\left(p_{i}\right), D_{ \pm}^{s}\left(p_{i}\right)$ the connected components of fundamental domains of $W^{u}\left(p_{i}\right), W^{s}\left(p_{i}\right)$, respectively. Let $d_{p_{i}}$ be a linearizing metric at $p_{i}$ and $\lambda_{i}, \mu_{i}$ be the eigenvalues of $D f\left(p_{i}\right)$ with $\left|\lambda_{i}\right|<1$ and $\left|\mu_{i}\right|>1$. Similarly, $d_{\bar{p}_{i}}, \bar{\lambda}_{i}, \bar{\mu}_{i}$ denote the corresponding objects for $\bar{f} \in \mathscr{B}$. Then we have

LEMMA 2.2. Let $f, \bar{f} \in \mathscr{B}$. Assume that $\left(p_{1}, p_{2}, p_{3}\right),\left(\bar{p}_{1}, \bar{p}_{2}, \bar{p}_{3}\right)$ are 3-chains of $f, \bar{f}$ such that $\left(p_{2}, p_{3}, \gamma_{2,3}\right),\left(\bar{p}_{2}, \bar{p}_{3}, \bar{\gamma}_{2,3}\right)$ are saddle connections. Suppose the following conditions are satisfied:

(1) $\log \left|\mu_{3}\right| / \log \left|\lambda_{2}\right|=\log \left|\bar{\mu}_{3}\right| / \log \left|\bar{\lambda}_{2}\right|$.

(2) There exists a homeomorphism $\Psi: \gamma_{2,3} \rightarrow \bar{\gamma}_{2,3}$ and a point $q \in \gamma_{2,3}$ such that $T_{f, q}=T_{\bar{f}, \bar{q}} \circ \Psi$, where $\bar{q}=\Psi(q)$.

(3) $\left[d_{\bar{p}_{2}}\left(\bar{x}_{i}, \bar{p}_{2}\right) /\left(d_{p_{2}}\left(x_{i}, p_{2}\right)\right)^{c}\right]=\left[d_{\bar{p}_{2}}\left(\bar{x}_{1}, \bar{p}_{2}\right) /\left(d_{p_{2}}\left(x_{1}, p_{2}\right)\right)^{c}\right]=$ a for $i=2, \ldots, l$, where $\left\{x_{1}, \ldots, x_{l}\right\}=W^{u}\left(p_{1}\right) \cap D_{-}^{s}\left(p_{2}\right),\left\{\bar{x}_{1}, \ldots, \bar{x}_{l}\right\}=W^{u}\left(\bar{p}_{1}\right) \cap D_{-}^{s}\left(\bar{p}_{2}\right)$ and $c=$ $\log \left|\bar{\lambda}_{2}\right| / \log \left|\lambda_{2}\right|$.

(4) $\lambda_{1}=\bar{\lambda}_{1}$ and

$$
\bar{c}_{i} d_{\bar{p}_{2}}\left(\bar{x}_{i}, \bar{p}_{2}\right) /\left(c_{i} d_{p_{2}}\left(x_{i}, p_{2}\right)\right)=\bar{c}_{1} d_{\bar{p}_{2}}\left(\bar{x}_{1}, p_{2}\right) /\left(c_{1} d_{p_{2}}\left(x_{1}, p_{2}\right)\right)=b
$$

for $i=1, \ldots, l$, where

$$
c_{i}=\lim _{\substack{x \rightarrow x_{i} \\ x \in W^{s}\left(p_{2}\right)}} \frac{d_{p_{1}}\left(x, x_{i}\right)}{d_{p_{2}}\left(x, x_{i}\right)}, \quad \bar{c}_{i}=\lim _{\substack{\bar{x} \rightarrow \bar{x}_{i} \\ \bar{x} \in W^{s}\left(\bar{p}_{2}\right)}} \frac{d_{\bar{p}_{1}}\left(\bar{x}, \bar{x}_{i}\right)}{d_{\bar{p}_{2}}\left(\bar{x}, \bar{x}_{i}\right)} .
$$

Then there exists a G-equivariant conjugacy $h: U \rightarrow \bar{U}$ between $f$ and $\bar{f}$ where $U$ (resp. $\bar{U})$ is a $G$ - and f-invariant ( $\bar{G}$ - and $\bar{f}$-invariant) open set containing $p_{1}, p_{2}$ and $p_{3}$ (resp. $\bar{p}_{1}, \bar{p}_{2}$ and $\bar{p}_{3}$ ).

Proof. Let $\mathscr{F}_{p_{1}}^{u}\left(\right.$ resp. $\left.\mathscr{F}_{\bar{p}_{1}}^{u}\right)$ be a $C^{1}$ unstable foliation at $p_{1}$ (resp. $\left.\bar{p}_{1}\right)$ and $\mathscr{F}_{p_{2}}^{u}$ (resp. $\mathscr{F}_{\bar{p}_{2}}^{u}$ ) be an unstable foliation at $p_{2}$ (resp. $\bar{p}_{2}$ ) compatible with $\mathscr{F}_{p_{1}}^{u}\left(\right.$ resp. $\left.\mathscr{F}_{\bar{p}_{1}}{ }^{u}\right)$ defined 
on a $G$ - and f-invariant neighborhood $V$ of $p_{2}$. Let $h: W^{s}\left(p_{2}\right) \rightarrow W^{s}\left(\bar{p}_{2}\right)$ be the map satisfying $h\left(x_{1}\right)=\bar{x}_{1}, h\left(p_{2}\right)=\bar{p}_{2}$ and

$$
\frac{d_{\bar{p}_{2}}\left(h(x), \bar{p}_{2}\right)}{\left[d_{p_{2}}\left(x, p_{2}\right)\right]^{c}}=\frac{d_{\bar{p}_{2}}\left(\bar{x}_{1}, \bar{p}_{2}\right)}{\left[d_{p_{2}}\left(x_{1}, p_{2}\right)\right]^{c}}
$$

for $x \neq p_{2}$. Clearly $h$ is a homeomorphism conjugating $f$ and $\bar{f}$. Since $T_{f, q}=T_{\bar{f}, \bar{q}} \circ \Psi$ and $\Psi(q)=\bar{q}$, there exists a homeomorphism $h: \gamma_{23} \rightarrow \bar{\gamma}_{23}$ such that $h(q)=\bar{q}$, $h \circ f=\bar{f} \circ h$ and $T_{f, q}=T_{\bar{f}, \bar{q}} \circ h$. We can extend this homeomorphism to a conjugacy on $W^{u}\left(p_{2}\right) \cup W^{s}\left(p_{3}\right)$.

Let $\mathscr{F}_{p_{2}}^{s}\left(\mathscr{F}_{p_{3}}^{u}\right)$ be a stable (unstable) foliation at $p_{2}\left(p_{3}\right)$ defined on an invariant neighborhood $V_{p_{2}} \subset V\left(V_{p_{3}}\right)$ so that $\mathscr{F}_{p_{2}}^{s}$ and $\mathscr{F}_{p_{3}}^{u}$ coincide in $V_{p_{2}} \cap V_{p_{3}}$. Using the foliations $\mathscr{F}_{p_{2}}^{s}$ and $\mathscr{F}_{p_{2}}{ }^{u}$ and the corresponding ones for $\bar{f}$ we extend $h$ continuously to a conjugacy on $V_{p_{2}}$. Now define $h: W^{u}\left(p_{3}\right) \rightarrow W^{u}\left(\bar{p}_{3}\right)$ so that

$$
\frac{d_{\bar{p}_{3}}\left(h(w), \bar{p}_{3}\right)}{\left[d_{p_{3}}\left(w, p_{3}\right)\right]^{c}}=\frac{\beta_{\bar{f}}(h(q))}{\left[\beta_{f}(q)\right]^{c}} \cdot \frac{d_{\bar{p}_{2}}\left(\bar{x}_{1}, \bar{p}_{2}\right)}{\left[d_{p_{2}}\left(x_{1}, p_{2}\right)\right]^{c}}
$$

for every $w \in W^{u}\left(p_{3}\right)$. Since the eigenvalue condition is satisfied $h$ is a conjugacy on $W^{u}\left(p_{3}\right)$. We claim that $h$ is continuous on $W^{u}\left(p_{3}\right)$. Indeed let $w_{n} \rightarrow w \in W^{u}\left(p_{3}\right)$, $w_{n} \in V_{p_{3}}$. Suppose by contradiction that $h\left(w_{n}\right)$ does not converge to $h(w)$. We may assume, by taking a subsequence if necessary, that no subsequence of $d_{\bar{p}_{3}}\left(h\left(w_{n}\right), \bar{\gamma}_{23}\right)$ converges to $d_{\bar{p}_{3}}\left(h(w), \bar{p}_{3}\right)$. By taking a subsequence we may assume there exist sequences $j_{n}, k_{n} \rightarrow+\infty$ such that $f^{-k_{n}}\left(w_{n}\right)=y_{n}$ converges to $y \in \gamma_{23}$ and $f^{-j_{n}}\left(y_{n}\right)$ $=z_{n}$ converges to $z \in W^{s}\left(p_{2}\right)$. Clearly $h\left(z_{n}\right) \rightarrow h(z)$ and $h\left(y_{n}\right) \rightarrow h(y)$. We have

$$
\frac{d_{\bar{p}_{2}}\left(h\left(z_{n}\right), \bar{\gamma}_{23}\right)}{\left[d_{p_{2}}\left(z_{n}, \gamma_{23}\right)\right]^{c}} \rightarrow \frac{d_{\bar{p}_{2}}\left(h(z), \bar{p}_{2}\right)}{\left[d_{p_{2}}\left(z, p_{2}\right)\right]^{c}} \text {. }
$$

Since $\left|\bar{\lambda}_{2}\right|=\left|\lambda_{2}\right|^{c}$ and $\left|\bar{\mu}_{3}\right|=\left|\mu_{3}\right|^{c}$,

$$
\frac{d_{\bar{p}_{2}}\left(h\left(z_{n}\right), \bar{\gamma}_{23}\right)}{\left[d_{p_{2}}\left(z_{n}, \gamma_{23}\right)\right]^{c}}=\frac{\left|\bar{\lambda}_{2}\right|^{-j_{n}}}{\left|\lambda_{2}\right|^{-c j_{n}}} \cdot \frac{d_{\bar{p}_{2}}\left(h\left(y_{n}\right), \bar{\gamma}_{23}\right)}{\left[d_{p_{2}}\left(y_{n}, \gamma_{23}\right)\right]^{c}}=\frac{d_{\bar{p}_{2}}\left(h\left(y_{n}\right), \bar{\gamma}_{23}\right)}{\left[d_{p_{2}}\left(y_{n}, \gamma_{23}\right)\right]^{c}}
$$

and then

$$
\begin{aligned}
\frac{d_{\bar{p}_{3}}\left(h\left(w_{n}\right), \bar{\gamma}_{23}\right)}{\left[d_{p_{3}}\left(w_{n}, \gamma_{23}\right)\right]^{c}} & =\frac{\left|\bar{\mu}_{3}\right|^{+k_{n}}}{\left|\mu_{3}\right|^{+c k_{n}}} \cdot \frac{d_{\bar{p}_{3}}\left(h\left(y_{n}\right), \bar{\gamma}_{23}\right)}{\left[d_{p_{3}}\left(y_{n}, \gamma_{23}\right)\right]^{c}}=\frac{d_{\bar{p}_{3}}\left(h\left(y_{n}\right), \bar{\gamma}_{23}\right)}{\left[d_{p_{3}}\left(y_{n}, \gamma_{23}\right)\right]^{c}} \\
& \rightarrow \frac{\beta_{f}(h(y))}{\left[\beta_{f}(y)\right]^{c}} \cdot \frac{d_{\bar{p}_{2}}\left(h(z), \bar{p}_{2}\right)}{\left[d_{p_{2}}\left(z, p_{2}\right)\right]^{c}}=\frac{\beta_{f}(h(q))}{\left[\beta_{f}(q)\right]^{c}} \cdot \frac{d_{\bar{p}_{2}}\left(h(z), \bar{p}_{2}\right)}{\left[d_{p_{2}}\left(z, p_{2}\right)\right]^{c}} .
\end{aligned}
$$

Hence

$$
d_{\bar{p}_{3}}\left(h\left(w_{n}\right), \bar{\gamma}_{23}\right) \rightarrow\left[d_{p_{3}}\left(w, p_{3}\right)\right]^{c} \cdot \frac{\beta_{\bar{f}}(h(q))}{\left[\beta_{f}(q)\right]^{c}} \cdot \frac{d_{\bar{p}_{2}}\left(h\left(x_{1}\right), \bar{p}_{2}\right)}{\left[d_{p_{2}}\left(x_{1}\right), p_{2}\right]^{c}}=d_{\bar{p}_{3}}\left(h(w), \bar{p}_{3}\right) .
$$

This is a contradiction and proves that $h\left(w_{n}\right)$ converges to $h(w)$.

We define $h$ on $W^{s}\left(p_{1}\right)$ as the map satisfying

$$
\frac{d_{\bar{p}_{1}}\left(h(x), \bar{p}_{1}\right)}{d_{p_{1}}\left(x, p_{1}\right)}=b \frac{c}{a} \quad \forall x \in W^{s}\left(p_{1}\right),
$$


where $b$ and $a$ are as in (3) and (4). Since $\lambda_{1}=\bar{\lambda}_{1}$ it follows that $h: W^{s}\left(p_{1}\right) \rightarrow W^{s}\left(\bar{p}_{1}\right)$ is a conjugacy. Let us prove the continuity of $h$ at $W^{s}\left(p_{1}\right)$. For that we consider a sequence

$$
w_{n} \rightarrow w \in W^{s}\left(p_{1}\right), \quad w_{n} \in V_{p_{2}} .
$$

Suppose by contradiction that $h\left(w_{n}\right)$ does not converge to $h(w)$. We may assume, by taking a subsequence if necessary, that no subsequence of $d_{\bar{p}_{1}}\left(h\left(w_{n}\right), W^{u}\left(\bar{p}_{1}\right)\right)$ converges to $d_{\bar{p}_{1}}\left(h(w), \bar{p}_{1}\right)$. For each $n$ let $y_{n} \in W^{s}\left(p_{2}\right)$ be the intersection with $W^{s}\left(p_{2}\right)$ of the leaf of $\mathscr{F}_{p_{2}}^{u}$ through $w_{n}$. By the compatibility of the foliations $\mathscr{F}_{p_{1}}{ }^{u}$ and $\mathscr{F}_{p_{2}}{ }^{u}$ it follows that $y_{n}$ and $w_{n}$ are in the same leaf of $\mathscr{F}_{p_{1}}^{u}$. Hence $y_{n} \rightarrow w$. Since $h$ maps leaves of $\mathscr{F}_{p_{2}}{ }^{u}$ into leaves of $\mathscr{F}_{\bar{p}_{2}}{ }^{u}$ and the foliations $\mathscr{F}_{\bar{p}_{1}}{ }^{u}$ and $\mathscr{F}_{\bar{p}_{2}}{ }^{u}$ are compatible it follows that no subsequence of $d_{\bar{p}_{1}}\left(h\left(y_{n}\right), W^{u}\left(\bar{p}_{1}\right)\right)$ converges to $d_{\bar{p}_{1}}\left(h(w), \bar{p}_{1}\right)$. By taking a subsequence we may assume that there exists a sequence $k_{n} \rightarrow \infty$ such that $f^{k_{n}}\left(y_{n}\right)$ converges to $x_{j}$ for some $j=1, \ldots, l$. Hence $\bar{f}^{k_{n}}\left(h\left(y_{n}\right)\right)$ converges to $\bar{x}_{j}$. On the other hand we have

$$
\begin{aligned}
\lim _{n \rightarrow \infty} d_{\bar{p}_{1}}\left(h\left(y_{n}\right), W^{u}\left(p_{1}\right)\right) & =\lim _{n \rightarrow \infty}\left|\bar{\lambda}_{1}\right|^{-k_{n}} d_{\bar{p}_{1}}\left(\bar{f}^{k_{n}}\left(h\left(y_{n}\right)\right), \bar{x}_{j}\right) \\
& =\lim _{n \rightarrow \infty}\left|\bar{\lambda}_{1}\right|^{-k_{n}} \bar{c}_{j} d_{\bar{p}_{2}}\left(\bar{f}^{k_{n}}\left(h\left(y_{n}\right)\right), \bar{x}_{j}\right) \\
& =\lim _{n \rightarrow \infty}\left|\bar{\lambda}_{1}\right|^{-k_{n}} \bar{c}_{j} c\left[d_{p_{2}}\left(x_{j}, p_{2}\right)\right]^{c-1} d_{p_{2}}\left(f^{k}\left(y_{n}\right), x_{j}\right) \\
& =\lim _{n \rightarrow \infty}\left|\bar{\lambda}_{1}\right|^{-k_{n}} \bar{c}_{j} c\left[d_{p_{2}}\left(x_{j}, p_{2}\right)\right]^{c-1} \frac{1}{c_{j}} d_{p_{1}}\left(f^{k}\left(y_{n}\right), x_{j}\right) \\
& =c \frac{\bar{c}_{j}}{c_{j}}\left[d_{p_{2}}\left(x_{j}, p_{2}\right)\right]^{c-1} d_{p_{1}}\left(w, p_{1}\right) \\
& =\frac{c \bar{c}_{j}}{c_{j}} \frac{1}{a} \frac{d_{\bar{p}_{2}}\left(\bar{x}_{j}, \bar{p}_{2}\right)}{d_{p_{2}}\left(x_{j}, p_{2}\right)} d_{p_{1}}\left(w, p_{1}\right) \\
& =\frac{c}{a} b d_{p_{1}}\left(w, p_{1}\right)=d_{\bar{p}_{1}}\left(h(w), \bar{p}_{1}\right) .
\end{aligned}
$$

This is a contradiction. Hence $h$ is continuous at $w$. Now we have to extend $h$ continuously to a conjugacy on a full neighborhood of $p_{1}$. Notice that the conjugacy we have already defined on $W^{s}\left(p_{1}\right) \cup V_{p_{2}}$ does not map leaves of $\mathscr{F}_{p_{1}}^{u}$ into leaves of $\mathscr{F}_{\bar{p}_{1}}{ }^{u}$. In fact, if $L_{x}$ is the leaf of $\mathscr{F}_{p_{1}}{ }^{u}$ through $x \in W^{s}\left(p_{1}\right)$, then each component of $L_{x} \cap V$ is mapped into a leaî of $\mathscr{F}_{\bar{p}_{1}}{ }^{u}$ but different components may be mapped into different leaves. On the other hand, it follows from the above computations that $h(x)$ is the only accumulation point of the images of these components. Then we can construct a continuous curve $\tilde{L}_{h(x)}$ through the point $h(x)$ by connecting two consecutive components of $h\left(L_{x} \cap V\right)$ by a curve whose expression in a coordinate system linearizing $f$ at $\bar{p}_{1}$ is a line segment. Therefore the family of continuous curves $\tilde{\mathscr{F}}_{\bar{p}_{1}} u=\left\{\tilde{L}_{h(x)} ; x \in W^{s}\left(p_{1}\right)\right\}$ is an $\bar{f}$-invariant foliation on a neighborhood of $\bar{p}_{1}$. Now we construct a stable foliation $\mathscr{F}_{p_{1}}^{s}\left(\right.$ resp. $\mathscr{F}_{\bar{p}_{1}}^{s}$ ) on a neighborhood $V_{p_{1}}$ of $p_{1}$ (resp. $V_{\bar{p}_{1}}$ of $\bar{p}_{1}$ ) such that each component of the intersection of the boundary of $V_{p_{2}}$ (resp. 
$\left.h\left(V_{p_{2}}\right)\right)$ with $V_{p_{1}}\left(\right.$ resp. $\left.V_{\bar{p}_{1}}\right)$ is a leaf of $\mathscr{F}_{p_{1}}^{s}\left(\operatorname{resp} . \mathscr{F}_{\bar{p}_{1}}^{s}\right)$. Next we extend $h \mid V_{p_{2}} \cap W^{u}\left(p_{1}\right)$ to a conjugacy on $W^{u}\left(p_{1}\right)$. There is a unique extension of $h$ to a conjugacy on an invariant neighborhood $V_{p_{1}}$ of $p_{1}$ mapping leaves of $\mathscr{F}_{p_{1}}^{u}$ into leaves of $\tilde{F}_{p_{1}}^{u}$ and leaves of $\mathscr{F}_{p_{1}}^{s}$ into leaves of $\mathscr{F}_{\bar{p}_{1}}^{s}$..

Now let $\mathscr{F}_{p_{3}}^{s}\left(\mathscr{F}_{\bar{p}_{3}}^{s}\right)$ be a stable foliation at $p_{3}\left(\bar{p}_{3}\right)$. Using these foliations and the unstable foliations $\mathscr{F}_{p_{3}}{ }^{u}, \mathscr{F}_{\bar{p}_{3}}{ }^{u}$ we extend $h$ to $V_{p_{3}}-V_{p_{2}}$.

All the above constructions can be done in the $G$-equivariant context.

In the next lemma we study the behavior of $G$ - and $f$-invariant unstable and stable foliations $\mathscr{F}_{p_{2}}^{u}, \mathscr{F}_{p_{3}}^{s}$ near a saddle connection $\left(p_{2}, p_{3}, \gamma_{23}\right)$ when its transition function is monotone.

LEMMA 2.3. Let $f \in \mathscr{B}$ and assume that $\left(p_{2}, p_{3}, \gamma_{23}\right)$ is a saddle connection of $f$. If $\mathscr{F}_{p_{2}}^{u}$ and $\mathscr{F}_{p_{3}}^{s}$ are $G$ - and f-invariant $C^{1}$ unstable and stable foliations at $p_{2}$ and $p_{3}$, respectively, and the transition function $T_{f, q}, q \in \gamma_{23}$, is monotone, then there is a $G$ and f-invariant neighborhood $U$ of $\gamma_{23}$, where $\mathscr{F}_{p_{2}}^{u}$ and $\mathscr{F}_{p_{3}}^{s}$ are $G$-transversal to each other.

Proof. Consider a fundamental neighborhood $N^{s} \supset[q, f(q)]$ of $W^{s}\left(p_{3}\right)$ and linearizing metrics $d_{p_{i}}$ at $p_{i}, i=2,3$. We may assume that with these metrics the distances to $\gamma_{23}$ are constant along the leaves of the corresponding foliations, and we may consider the leaves of both foliations only in one side of $\gamma_{23}$.

Let $\mathscr{F}_{p_{3}}^{u}$ be a $C^{1}$ unstable foliation at $p_{3}$. We define $\alpha_{x}(y)=\alpha(x, y)=d_{p_{3}}\left(z, \gamma_{23}\right)$, where $z$ is the intersection point of the $\mathscr{F}_{p_{2}}^{u}$-leaf through $x \in W^{s}\left(p_{2}\right)$ with the $\mathscr{F}_{p_{3}}{ }^{u}$-leaf through $y \in[q, f(p)] \subset \gamma_{23}$. By the hypothesis $\alpha$ is a well-defined $C^{1}$ function if we take $x \in W^{s}\left(p_{2}\right)$ sufficiently near $p_{2}$. We will identify $x$ with $d_{p_{2}}\left(x, p_{2}\right)$. Then it is enough to prove that $\alpha_{x}^{\prime}(y) \neq 0$ for all $y \in[q, f(q)]$ and all $x$ sufficiently small. We have

$$
\lim _{x \rightarrow 0} \frac{\alpha(x, y)}{x}=\lim _{z \rightarrow y} \frac{d_{p_{3}}\left(z, \gamma_{23}\right)}{d_{p_{2}}\left(z, \gamma_{23}\right)}=\beta_{f}(y) .
$$

But $\alpha(0, y)=0$. Then $\alpha(x, y)=x g(x, y)$, where $g$ is a $C^{1}$ function such that $g(0, y)=\beta_{f}(y)$. Now the lemma follows from the fact that $T_{f, q}$ is monotone if and only if $\beta_{f}^{\prime}(y) \neq 0$ for all $y \in[q, f(q)]$.

REMARK. With the above notation, if $f \in \mathscr{B}, T_{f, q}$ has a (nondegenerate) critical point $y \in[q, f(q)]$, then there is a $C^{1}$ curve of tangencies between $\mathscr{F}_{p_{2}}^{u}$ and $\mathscr{F}_{p_{3}}^{u}$ containing $y$.

Now we are going to use (2.3) to construct a conjugacy between $f$ and $\bar{f}, f, \bar{f} \in \mathscr{B}$, near saddle connections.

LEMMA 2.4. Let $f, \bar{f} \in \mathscr{B}$ and assume that $\left(p_{2}, p_{3}, \gamma_{23}\right)$ and $\left(\bar{p}_{2}, \bar{p}_{3}, \bar{\gamma}_{23}\right)$ are saddle connections of $f$ and $\bar{f}$. Let $\mathscr{F}_{p_{2}}^{u}$ and $\mathscr{F}_{p_{3}}^{s}\left(\mathscr{F}_{\bar{p}_{2}}^{u}\right.$ and $\left.\mathscr{F}_{\bar{p}_{3}}^{s}\right)$ be $G$ - and $f-(\bar{f})$-invariant unstable and stable foliations at $p_{2}$ and $p_{3}\left(\bar{p}_{2}\right.$ and $\left.\bar{p}_{3}\right)$ respectively. If the eigenvalue condition is satisfied and the transition functions $T_{f, q}, T_{\bar{f}, \bar{q}}, q \in \gamma_{23}, \bar{q} \in \bar{\gamma}_{23}$ have no critical points, then there is a G-equivariant conjugacy $h$ beiween $f$ and $\bar{f}$ in neighborhoods of $\mathrm{cl}\left(\gamma_{23}\right)$ and $\mathrm{cl}\left(\bar{\gamma}_{23}\right)$, sending leaves of $\mathscr{F}_{p_{2}}^{u}$ and $\mathscr{F}_{p_{3}}^{s}$ on leaves of $\mathscr{F}_{\bar{p}_{2}}^{u}$ and $\mathscr{F}_{\bar{p}_{3}}^{s}$, respectively, and $h(q)=\bar{q}$. 
Proof. By the hypothesis we can define a conjugacy

$$
h: \bigcup_{i=2}^{3}\left[W^{s}\left(p_{i}\right) \cup W^{u}\left(p_{i}\right)\right] \rightarrow \bigcup_{i=2}^{3}\left[W^{s}\left(\bar{p}_{i}\right) \cup W^{u}\left(\bar{p}_{i}\right)\right]
$$

between $f$ and $\bar{f}$ in exactly the same way as we did in the proof of (2.2). In particular $h(q)=\bar{q}$.

Let $U(\bar{U})$ be the $G$ - and $f-(\bar{f})$-invariant neighborhood of $\gamma_{23}\left(\bar{\gamma}_{23}\right)$, where $\mathscr{F}_{p_{3}}^{s}$ and $\mathscr{F}_{p_{2}}^{u}\left(\mathscr{F}_{\bar{p}_{3}}^{s}\right.$ and $\left.\mathscr{F}_{\bar{p}_{2}}^{u}\right)$ are $G$-transversal to each other given by (2.3). Using these transversal foliations, we extend $h$ to a conjugacy between $f$ and $\bar{f}$ from $\operatorname{cl}(U)$ to $\operatorname{cl}(\bar{U})$. The continuity of $h$ in $\gamma_{23}$ follows from (1.6).

Using those foliations, stable foliations at $p_{2}$ and $\bar{p}_{2}$, and unstable foliations at $p_{3}$ and $\bar{p}_{3}$, we extend $h$ to a neighborhood of $c l\left(\gamma_{23}\right)$.

All the above constructions can be done in the $G$-equivariant context.

In the next lemmas we study the remaining cases of infinite modulus of stability. We start considering the behavior of invariant foliations near saddle connections whose transition functions have critical points. We have

LEMMA 2.5. Let $f \in \mathscr{B}$ and assume that $\left(p_{2}, p_{3}, \gamma_{23}\right)$ is a saddle connection of $f$. Let $\Sigma$ be a small $C^{\infty}$ arc transverse to $W^{s}\left(p_{2}\right), \tilde{\Sigma}=\bigcup_{n \in \mathbf{Z}} f^{n}(\Sigma), \mathscr{F}_{p_{3}}^{s}$ a $C^{1}$ stable foliation at $p_{3}$ and $\pi^{s}: U_{p_{3}} \rightarrow W^{u}\left(p_{3}\right)$ the projection along the leaves of $\mathscr{F}_{p_{3}}^{s}$, where $U_{p_{3}}$ is a neighborhood of $W^{u}\left(p_{3}\right)$. Then $\left.\pi^{s}\right|_{\Sigma}$ has critical points if and only if $T_{f, q}, q \in \gamma_{23}$, has a critical point. Moreover, if $w \in W^{u}\left(p_{3}\right)$ is in the same side of $\gamma_{23}$ as $\Sigma$ and $\log \left|\mu_{3}\right| / \log \left|\lambda_{2}\right|$ is irrational, then there is a sequence $\left\{w_{n}\right\} \subset \tilde{\Sigma}$ of critical points of $\left.\pi^{s}\right|_{\Sigma}$ such that $w_{n} \rightarrow w$.

Proof. The first part of the lemma follows from the remark after (2.3). Notice that in fact we can construct a sequence $\left\{z_{n}\right\} \subset \tilde{\Sigma}$ of critical points of $\left.\pi^{s}\right|_{\tilde{\Sigma}}$ such that $z_{n} \rightarrow z$, with $z \in \gamma_{23}$.

For the second part, let $\left\{j_{n}\right\}$ and $\left\{k_{n}\right\}$ be sequences of positive integers such that $j_{n} \rightarrow+\infty, k_{n} \rightarrow+\infty$, and $f^{-j_{n}}\left(z_{n}\right) \in \Sigma, z_{n}$ as above, and $d_{p_{i}}$ is a linearizing metric at $p_{i}, i=2,3$. Let $x_{n}=f^{-j_{n}}\left(z_{n}\right)$ and $w_{n}=f^{k_{n}}\left(z_{n}\right)$. We have $d_{p_{3}}\left(w_{n}, \gamma_{23}\right)=$ $c_{n}\left|\lambda_{2}\right|^{j_{n}}\left|\mu_{3}\right|^{k_{n}}$, where

$$
c_{n}=\frac{d_{p_{3}}\left(z_{n}, \gamma_{23}\right)}{d_{p_{2}}\left(z_{n}, \gamma_{23}\right)} \cdot d_{p_{2}}\left(x_{n}, \gamma_{23}\right)
$$

Then $\log \left[d_{p_{3}}\left(w_{n}, \gamma_{23}\right)\right]=\log c_{n}+j_{n} \log \left|\lambda_{2}\right|+k_{n} \log \left|\mu_{3}\right|$. Since $\log c_{n}$ converges and $\log \left|\mu_{3}\right| / \log \left|\lambda_{2}\right|$ is irrational, given $w \in W^{u}\left(p_{3}\right)$ in the same side of $\gamma_{23}$ as $\Sigma$ it is possible to choose $\left\{j_{n}\right\}$ and $\left\{k_{n}\right\}$ such that $\log \left[d_{p_{3}}\left(w_{n}, \gamma_{23}\right)\right] \rightarrow d_{p_{3}}\left(w, p_{3}\right)$, and then $w_{n} \rightarrow w$, finishing the proof of the lemma.

LEMMA 2.6. Let $f \in \mathscr{B}$ and assume that $\left(p_{1}, p_{2}, p_{3}, p_{4}\right)$ is a 4-chain of saddles of $f$ which has a unique saddle connection $\left(p_{2}, p_{3}, \gamma_{23}\right)$. If $T_{f, q}, q \in \gamma_{23}$, has a critical point, then $f$ has infinite modulus of stability. 
Proof. Let $\Sigma \subset W^{u}\left(p_{1}\right)$ be a small arc intersecting $W^{s}\left(p_{2}\right)$ transversally at a unique point. From (1.7) we can build a $C^{1}$ stable foliation $\mathscr{F}_{p_{3}}$ in a neighborhood $U_{p_{3}}$ of $W^{u}\left(p_{3}\right)$ such that $W^{s}\left(p_{4}\right) \cap U_{p_{3}}$ is a union of leaves. Let $\pi^{s}: U_{p_{3}} \rightarrow W^{u}\left(p_{3}\right)$ be the projection along the leaves of $\mathscr{F}_{p_{3}}^{s}$. By the $G$-equivariance of $f$ we can choose

$$
w \in W^{u}\left(p_{3}\right) \cap W^{s}\left(p_{4}\right)
$$

in the same side of $\gamma_{23}$ as $\Sigma$. We may assume that $\log \left|\mu_{3}\right| / \log \left|\lambda_{2}\right|$ is irrational. Let $w_{n} \rightarrow w$ be a sequence of critical points of $\left.\pi^{s}\right|_{\Sigma}$ given by (2.5). The points $w_{n}$ are tangency points between $W^{u}\left(p_{1}\right)$ and leaves of $\mathscr{F}_{p_{3}}^{s}$. Thus, by small $C^{r}$ perturbations of $f$, supported in a small neighborhood of $w$ we can create as many tangency orbits between $W^{u}\left(p_{1}\right)$ and $W^{s}\left(p_{4}\right)$ as we want. Then, by (1.5), $f$ has infinite modulus of stability.

The proof of this lemma shows that $G$-transversality is not an open property in Diff $_{G}(M)$ even for elements in $\mathscr{B}$.

LEMMA 2.7. (a) Let $f \in \mathscr{B}$ and assume that $\left(p_{1}, p_{2}, p_{3}, p_{4}\right)$ is a 4-chain of saddles of $f$ whose unique saddle connections are $\left(p_{1}, p_{2}\right)$ and $\left(p_{3}, p_{4}\right)$. Then the modulus of stability of $f$ is infinite.

(b) Let $f \in \mathscr{B}$ and assume that $\left(p_{1}, p_{2}, p_{3}\right)$ is a 3-chain of saddles of $f$ such that $\left(p_{1}, p_{2}\right)$ and $\left(p_{2}, p_{3}\right)$ are saddle connections. Then the modulus of stability of $f$ is infinite.

Proof. Part (b) of this lemma is a simple consequence of (1.4).

We are going to prove (a). Let $h$ be a conjugacy between $f$ and $\bar{f} \in \mathscr{B}$, and $h\left(p_{i}\right)=\bar{p}_{i}, i=1,2,3,4$. From (1.4) it follows that $h: W_{+}^{u}\left(p_{2}\right) \rightarrow W_{+}^{u}\left(\bar{p}_{2}\right)$ is $C^{1}$, where $W_{+}^{u}\left(p_{2}\right)\left(W_{+}^{u}\left(\bar{p}_{2}\right)\right)$ is a connected component of $W^{u}\left(p_{2}\right)-\left\{p_{2}\right\}\left(W^{u}\left(\bar{p}_{2}\right)-\right.$ $\left\{\bar{p}_{2}\right\}$ ) such that $W_{+}^{u}\left(p_{2}\right) \cap W^{s}\left(p_{3}\right) \neq \varnothing$ (and so $\left.W_{+}^{u}\left(\bar{p}_{2}\right) \cap W^{s}\left(\bar{p}_{3}\right) \neq \varnothing\right)$. Let $d_{p_{3}}$ and $d_{\bar{p}_{3}}$ be linearizing metrics at $p_{3}$ and $\bar{p}_{3}$, and $z \in W_{+}^{u}\left(p_{2}\right) \cap W^{s}\left(p_{3}\right)$. Let $w \in W^{u}\left(p_{3}\right)$. By the $\lambda$-lemma there are sequences $\left\{z_{n}\right\},\left\{w_{n}\right\} \subset W_{+}^{u}\left(p_{2}\right)$ and $j_{n} \rightarrow+\infty$ such that $z_{n} \rightarrow z, w_{n} \rightarrow w$ and $w_{n}=f^{j_{n}}\left(z_{n}\right)$. Let $h\left(z_{n}\right)=\bar{z}_{n}, h\left(w_{n}\right)=\bar{w}_{n}$, $h(z)=\bar{z}$ and $h(w)=\bar{w}$. We have $d_{p_{3}}\left(w_{n}, W^{s}\left(p_{3}\right)\right)=\mu_{3}^{j_{n}} d_{p_{3}}\left(z_{n}, W^{s}\left(p_{3}\right)\right)$ and $d_{\bar{p}_{3}}\left(\bar{w}_{n}, W^{s}\left(\bar{p}_{3}\right)\right)=\bar{\mu}_{3}^{j_{n}} \cdot d_{\bar{p}_{3}}\left(\bar{z}_{n}, W^{s}\left(\bar{p}_{3}\right)\right)$. Since $d_{\bar{p}_{3}}\left(\bar{w}_{n}, W^{s}\left(\bar{p}_{3}\right)\right) \rightarrow d_{\bar{p}_{3}}\left(\bar{w}, \bar{p}_{3}\right)$, $d_{p_{3}}\left(w_{n}, W^{s}\left(p_{3}\right)\right) \rightarrow d_{p_{3}}\left(w, p_{3}\right)$ and $d_{\bar{p}_{3}}\left(\bar{z}_{n}, W^{s}\left(\bar{p}_{3}\right)\right) / d_{p_{3}}\left(z_{n}, W^{s}\left(p_{3}\right)\right) \rightarrow$ $\left(h \mid W_{+}^{u}\left(p_{2}\right)\right)^{\prime}(z)=a$, it follows that $\mu_{3}=\bar{\mu}_{3}$ and $d_{\bar{p}_{3}}\left(\bar{w}, \bar{p}_{3}\right) / d_{p_{3}}\left(w, p_{3}\right)=a$ for every $w \in W^{u}\left(p_{3}\right)$. This implies clearly that $f$ has infinite modulus of stability (see (1.3)).

REMARK. Let $f, \bar{f} \in \mathscr{B}$ and assume that $\left(p_{1}, p_{2}, p_{3}\right),\left(\bar{p}_{1}, \bar{p}_{2}, \bar{p}_{3}\right)$ are 3-chains of saddles of $f, \bar{f}$ such that $\left(p_{1}, p_{2}\right),\left(\bar{p}_{1}, \bar{p}_{2}\right)$ are their unique saddle connections. It follows from the proof of (2.7) that if $h$ is a conjugacy between $f$ and $\bar{f}$ such that $h\left(p_{i}\right)=\bar{p}_{i}, i=1,2,3$, then $\mu_{3}=\bar{\mu}_{3}$ and $h_{1} w^{u}\left(p_{3}\right)$ is well defined up to a real multiplicative constant.

Our final lemma of this section, which will be stated without proof, gives a case of finite modulus of stability. Its proof is a kind of globalization of the procedures used in the proof of (2.4), taking in account the remark after (1.3). 

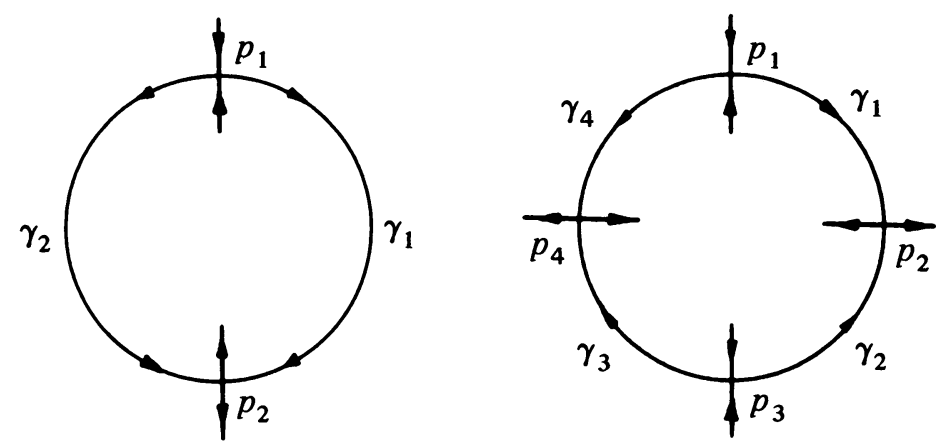

Figure 2

LEMMA 2.8. Let $f \in \mathscr{B}$ and assume that $p_{1}, \ldots, p_{2 l}, p_{2 l+1}=p_{1}$ are saddles of $f$ such that $\left(p_{i}, p_{i+1}, \gamma_{i}\right)$ is a saddle connection if $i$ is odd and $\left(p_{i+1}, p_{i}, \gamma_{i}\right)$ is a saddle connection if $i$ is even, $i=1, \ldots, 2 l+1$, as shown in Figure 2 . Let $\Gamma$ be the circle $\bigcup_{i=1}^{2 l}\left[c l\left(\gamma_{i}\right)\right]$ and $s_{i}$ be the number of orbits of critical points of the transition function associated to $\gamma_{i}$. The modulus of stability of $f$ in a neighborhood of $\Gamma$ is $\max \left\{1, s_{1}+s_{2}\right\}$ when $l=1$, and if $l>1$ it is $2 l+\left[\sum_{i=1}^{2 l}\left(s_{i}-1\right)\right]+1$ when $s_{i} \neq 0$ for all $i=1, \ldots, 2 l$, and $2 l+\left[\sum_{i=1}^{2 l} \max \left\{0, s_{i}-1\right\}\right]$ when $s_{i}=0$ for some $i \in\{1, \ldots, 2 l\}$.

END OF THE PROOF OF THE THEOREM. By the previous lemmas, it remains to prove that the elements in $\mathscr{C}$ have finite moduli of stability. Let $f_{0} \in \mathscr{C}$ and $\mathscr{N}$ be a small neighborhood of $f_{0}$. For $f, \bar{f} \in \mathscr{N}$ let $\operatorname{Per}(f)=\left\{p_{i}\right\}, \operatorname{Per}(\bar{f})=\left\{\bar{p}_{i}\right\}$, where $\bar{p}_{i}$ is near $p_{i}$ for each $i$. By the $G$-equivariance it follows that $\left(p_{i}, p_{i+1}, \gamma_{i}\right)$ is a saddle connection for $f$ if and only if $\left(\bar{p}_{i}, \bar{p}_{i+1}, \bar{\gamma}_{i}\right)$ is a saddle connection for $\bar{f}$. Suppose that the following conditions are satisfied:

(1) $f$ and $\bar{f}$ satisfy the eigenvalue condition for each corresponding saddle connection $\left(p_{i}, p_{i+1}, \gamma_{i}\right),\left(\bar{p}_{i}, \bar{p}_{i+1}, \bar{\gamma}_{i}\right)$.

(2) For each saddle connection $\gamma_{i}$ there exist $q_{i} \in \gamma_{i}$ and $\bar{q}_{i} \in \bar{\gamma}_{i}$ such that the transition functions $T_{f, q_{i}}, T_{\tilde{f}_{, \bar{q}_{i}}}$ have the same critical value and thus $T_{\bar{f}_{,}, \bar{q}_{i}} \circ \Psi_{i}=T_{f, q_{i}}$ for some homeomorphism $\Psi_{i}: \gamma_{i} \rightarrow \bar{\gamma}_{i}$.

(3) Let $\left(p_{i}, p_{i+1}, \gamma_{i}\right)$ be a saddle connection of $f$ and $q_{j}, j=1, \ldots, k$, be the saddle points whose unstable manifolds intersect $W^{s}\left(p_{i}\right)$. Let $\left\{x_{j, 1}, \ldots, x_{j, l(j)}\right\}=W^{u}\left(q_{j}\right)$ $\cap D_{+}^{s}\left(p_{i}\right)$, where $D_{+}^{s}\left(p_{i}\right)$ is a connected component of a fundamental domain for $W^{s}\left(p_{i}\right)$. Similarly $\left\{\bar{x}_{j, 1}, \ldots, \bar{x}_{j, l(j)}\right\}=W^{u}\left(\bar{q}_{j}\right) \cap D_{+}^{s}\left(p_{i}\right)$ with $\bar{q}_{j}$ near $q_{j}$ and $\bar{x}_{j, m}$ near $x_{j, m}$. Then the contracting eigenvalue of $D f_{q_{j}}$ is equal to the contracting eigenvalue of $D \bar{f}_{\bar{q}_{j}}$ and there exists a positive constant $a$ such that

$$
\frac{d_{\bar{p}_{i}}\left(\bar{x}_{j, m}, \bar{p}_{i}\right)}{\left[d_{p_{i}}\left(x_{j, m}, p_{i}\right)\right]^{c}}=a \text { for } m=1, \ldots, l(j), j=1, \ldots, k
$$

Here $d_{\bar{p}_{i}}$ is a linearizing metric at $\bar{p}_{i}$ and $d_{p_{i}}$ is a linearizing metric at $p_{i}$. 
(4) For each $j$ such that $l(j)>1$ let

$$
\begin{aligned}
& C_{j, n}=\lim _{\substack{x \rightarrow x_{j, n} \\
x \in W^{s}\left(p_{i}\right)}} \frac{d_{q_{j}}\left(x, x_{j, n}\right)}{d_{p_{i}}\left(x, x_{j, n}\right)}, \quad n=1, \ldots, l(j), \\
& \bar{C}_{j, n}=\lim _{\substack{\bar{x} \rightarrow \bar{x}_{j, n} \\
\bar{x} \in W^{s}\left(\bar{p}_{i}\right)}} \frac{d_{\bar{q}_{j}}\left(\bar{x}, \bar{x}_{j, n}\right)}{d_{\bar{p}_{i}}\left(x, x_{j, n}\right)}, \quad n=1, \ldots, l(j),
\end{aligned}
$$

where $d_{q_{j}}$ is a linearizing metric at $q_{j}$ and $d_{\bar{q}_{j}}$ is a linearizing metric at $\bar{q}_{j}$.

Then there exists a positive real number $b$ such that

$$
\frac{\bar{C}_{j, n}}{C_{j, n}} \cdot \frac{d_{\bar{p}_{i}}\left(\bar{x}_{j, n}, \bar{p}_{i}\right)}{d_{p_{i}}\left(x_{j, n}, p_{i}\right)}=b \quad \text { for all } n=1, \ldots, l(j) .
$$

(5) The statements dual of (3) and (4) concerning saddle points whose stable manifolds intersect $W^{u}\left(p_{i+1}\right)$ are satisfied.

We will prove that $f$ and $\bar{f}$ are conjugate. On the other hand, it follows from arguments in [dM and $\mathbf{d M}-\mathbf{v S}]$ that if $f$ and $\bar{f}$ are conjugate and $\left(\log \left|\mu_{i+1}\right|\right) /\left(\log \left|\lambda_{i}\right|\right)$ is irrational for each saddle connection $\left(p_{i}, p_{i+1}, \gamma_{i}\right)$, then the conditions (1)-(5) hold.

The construction of a conjugacy $h$ between $f$ and $\bar{f}$ is a globalization of the situations described in (2.2), (2.4) and (2.8) together with the construction of conjugacies between two Morse-Smale diffeomorphisms. The more difficult cases occur when there exist a saddle connection $\left(p_{i}, p_{i+1}, \gamma_{i}\right)$ which belongs to a 4-chain. Namely, there are saddle points $q_{1}, \ldots, q_{k}, r_{1}, \ldots, r_{k}$, such that $W^{u}\left(q_{j}\right)$ intersects transversally $W^{s}\left(p_{i}\right)$ and $W^{s}\left(r_{d}\right)$ intersects transversally $W^{u}\left(p_{i+1}\right)$. We will outline the construction of the conjugacy in this case. For details see the careful study of similar situations in [dM-vS]. We start by constructing for each saddle point $x$ of $f$ a $C^{1}$ unstable foliation $\mathscr{F}_{x}{ }^{u}$ satisfying the following compatibility condition: if $W^{u}(x)$ $\cap W^{s}(y) \neq \varnothing$ and $x$ does not belong to a saddle connection, then a leaf of $\mathscr{F}_{y}{ }^{u}$ is contained in a leaf of $\mathscr{F}_{x}{ }^{u}$ whenever they intersect each other. For this we proceed, as in [P2 and P-S], by induction on the phase-diagram of $f$ starting at saddles which do not belong to a saddle connection and whose stable manifolds are contained in unstable manifolds of sources. The construction proceeds exactly as in [P2] except when we arrive at a saddle connection $\left(p_{i}, p_{i+1}, \gamma_{i}\right)$. Then we construct an unstable foliation $\mathscr{F}_{p_{i}}{ }^{u}$ compatible with all the unstable foliations of saddles whose unstable manifolds intersect $W^{s}\left(p_{i}\right)$. If there is a saddle $r$ whose stable manifold intersects $W^{u}\left(p_{i+1}\right)$ we have by the transversality condition and Lemma 2.3 that the leaves of $\mathscr{F}_{p}{ }^{u}$ are transversal to $W^{s}(r)$. From this it follows that we can construct an unstable foliation $\mathscr{F}_{r}{ }^{u}$ which is compatible with $\mathscr{F}_{p_{i}}{ }^{u}$ and with all other unstable foliations of saddles whose stable manifolds intersect $W^{s}(r)$. Similarly we construct a system of compatible stable foliations $\mathscr{F}_{x}^{s}$ for each saddle $x$ which does not belong to a saddle connection. Let $\mathscr{F}_{\bar{x}}{ }^{u}$ and $\mathscr{F}_{\bar{x}}{ }^{x}$ denote the corresponding invariant foliations for $\bar{f}$, where $\bar{x}$ always denotes the saddle point of $\bar{f}$ near $x$. As in Lemma 2.2 we construct a conjugacy $h$ on an $f$-invariant neighborhood $V$ of $\gamma_{i}$ such that $h$ preserves the above 
foliations, map the unstable manifold of $q_{j}$ into the unstable manifold of $\bar{q}_{j}$ and the stable manifold of $r_{k}$ into the stable manifold of $\bar{r}_{k}$. As in Lemma 2.2 this extends continuously to conjugacy of $V \cup W^{s}\left(q_{j}\right) \cup W^{u}\left(r_{k}\right)$. We proceed by extending $h$ to $W^{u}\left(q_{j}\right)$ and $W^{s}\left(q_{j}\right)$ and $W^{s}\left(r_{k}\right)$. In order to be able to globalize the construction we have to be careful here. We define as in [P2] the conjugacy on every unstable manifold of saddles whose stable manifold intersects $W^{u}\left(q_{j}\right)$ so that $h$ preserves the stable foliations. This fixes a definition of $h$ on an open set of the fundamental domain of $W^{u}\left(q_{j}\right)$. We then define $h: W^{s}\left(q_{j}\right) \rightarrow W^{s}\left(\bar{q}_{j}\right)$ also extending this map. Using this and the arguments in Lemma 2.5 we extend $h$ to an invariant neighborhood of $q_{j}$. Similarly $h$ extends to an invariant neighborhood of $r_{k}$. After performing this construction for every saddle connection we can extend $h$ to $M$ as in [P2]. This finishes the proof.

3. The principal orbits have dimension $d>0$. Suppose the principal $G$-orbits have dimension one. Then the orbit space $M^{*}=M / G$ is diffeomorphic to $S^{1}$ or to the closed interval $[0,1]$. Any equivariant diffeomorphism $f: M \rightarrow M$ induces a diffeomorphism $f^{*}: M^{*} \rightarrow M^{*}$. Furthermore, given a neighborhood $\mathscr{N} \subset \operatorname{Diff}_{G}(M)$ of $f$ there exists a neighborhood $\mathscr{N}^{*}$ of $f^{*}$ such that for each $g^{*} \in \mathscr{N}^{*}$ there is a $g \in \mathscr{N}$ whose induced diffeomorphism is $g^{*}$. From this we get the following

Proposition 3.1. If the principal orbits have dimension 1 , then the set $\mathscr{A}$ of equivariant diffeomorphisms whose nonwandering set is a finite number of normally hyperbolic critical elements is open and dense in $\operatorname{Diff}_{G}(M)$.

If $f$ is an equivariant diffeomorphism then the isotropy group is constant along an $f$-orbit. Hence if $C$ is a $G$-orbit which is $f$-invariant, then $\left\{f^{n}(x) ; n \in \mathbf{Z}\right\} \subset N\left(G_{x}\right) \cdot x$ $=\left\{g x, g \in N\left(G_{x}\right)\right\}$, where $N\left(G_{x}\right)$ is the normalizer of the isotropy group $G_{x}$. Since $N\left(G_{x}\right) \cdot x$ is diffeomorphic to $N\left(G_{x}\right) / G_{x}$ we have that all points of $C$ are periodic whenever the dimension of $N\left(G_{x}\right) / G_{x}$ is zero. From the slice theorem [Br] it follows that if $\operatorname{dim} N\left(G_{x}\right) / G_{x}=0$ for some $x$ in a principal orbit, then $\operatorname{dim} N\left(G_{y}\right) / G_{y}=0$ for all $y$. In this case we also have that the set of $y \in M$ such that $G_{x}=G_{y}$ is a one-dimensional manifold which intersects transversally each one-dimensional $G$ orbit. This defines a one-dimensional foliation $F$ on $\left\{x \in M ; G_{x} \neq G\right\}$ which is invariant by any $f \in \operatorname{Diff}_{G}(M)$. If $\sigma$ is an attracting (resp. repelling) critical element of $f \in A$, then the foliation $\mathscr{F}_{\sigma}^{s s}$ (resp. $\mathscr{F}_{\sigma}{ }^{u u}$ ) is the restriction of $\mathscr{F}$ to $W^{s}(\sigma)$ (resp. $\left.W^{u}(\sigma)\right)$. Using this fact it is easy to prove the following

Proposition 3.2. If the dimension of $N\left(G_{x}\right) / G_{x}$ is zero for some $x$ in a principal orbit, then every $f \in \mathscr{A}$ is structurally stable.

Suppose now that $\operatorname{dim} N\left(G_{x}\right) / G_{x}=1$ for some $x \in M$. From the slice theorem it follows that the isotropy group is constant on the principal stratum. Let $\mathscr{B}$ be the set of $f \in \mathscr{A}$ such that if $\sigma_{1}, \sigma_{2}$ are one-dimensional critical elements of $f$ such that $W^{u}\left(\sigma_{1}\right) \cap W^{s}\left(\sigma_{2}\right) \neq \varnothing$, then $\mathscr{F}_{\sigma_{1}}^{u u}$ is transversal to $\mathscr{F}_{\sigma_{2}}^{s s}$ except along a countable 
number of $G$-orbits where the tangency between the corresponding leaves is parabolic. Then we have

THEOREM 3.3. If $\operatorname{dim}\left(N\left(G_{x}\right) / G_{x}\right)=1$ for some $x \in M$, then every $f \in \mathscr{B}$ has finite modulus.

Proof. Let $H$ be the isotropy group of a point $x$ in a principal orbit. Since the isotropy group is constant on the principal stratum it follows that $N(H) / H$ acts in $M$ and this action has the same $G$-orbit structure as that of $G$. Furthermore every $(N(H) / H)$-equivariant diffeomorphism is $G$-equivariant and vice versa. Hence we may assume that the isotropy group is trivial for every point in a principal orbit.

Let $\sigma_{i}, i=1,2$, be critical elements of $f \in \mathscr{A}$ such that $W^{u}\left(\sigma_{1}\right) \cap W^{s}\left(\sigma_{2}\right) \neq \varnothing$. Let $x_{0}$ be a point of nontransversal intersection between $W^{u u}\left(p_{1}\right)$ and $W^{s s}\left(p_{2}\right)$ for some $p_{i} \in \sigma_{i}$ (if there is no such point we take $x_{0}$ to be any point in $W^{u u}\left(p_{1}\right) \cap$ $\left.W^{s s}\left(p_{2}\right)\right)$. Let $C_{0}$ be the $G$-orbit of $x_{0}$ and $A \subset W^{u}\left(\sigma_{1}\right) \cap W^{s}\left(\sigma_{2}\right)$ be the cylinder bounded by $C_{0}$ and $f\left(C_{0}\right)$. The intersection with $A$ of the set of tangencies between $\mathscr{F}_{\sigma_{1}}^{u u}$ and $\mathscr{F}_{\sigma_{2}}^{s s}$ is a finite number of $G$-orbits $C_{0}, C_{1}, \ldots, C_{k}=f\left(C_{0}\right)$. Let $x_{i}=$ $W^{u u}\left(p_{1}\right) \cap C_{i}, y_{i}=W^{s s}\left(p_{2}\right) \cap C_{i}$ and $g_{i} \in G$ be the unique element such that $g_{i} y_{i}=x_{i}$ (here $x_{0}=y_{0}$ and $g_{0}=\mathrm{id}$ ). Let $\bar{f}$ be an equivariant diffeomorphism near $f$, $\bar{\sigma}_{i}$ be the critical element of $\bar{f}$ near $\sigma_{i}$ and $\bar{C}_{0}, \ldots, \bar{C}_{k}=\bar{f}\left(\bar{C}_{0}\right)$ be the $G$-orbits of tangencies between $\mathscr{F}_{\bar{\sigma}_{1}}^{u u}$ and $\mathscr{F}_{\bar{\sigma}_{2}}^{s s}$ with $\bar{C}_{i}$ near $C_{i}$. Let $\bar{x}_{i}=W^{u u}\left(\bar{p}_{1}\right) \cap \bar{C}_{i}, \bar{y}_{i}=$ $W^{s s}\left(\bar{p}_{2}\right) \cap \bar{C}_{i}$ and $\bar{g}_{i} \in G$ be such that $\bar{g}_{i} \bar{y}_{i}=\bar{x}_{i}$. Let $v_{j}, \bar{v}_{j} \in G, j=1,2$, be such that $f\left(p_{j}\right)=v_{j} p_{j}$ and $\bar{f}\left(\bar{p}_{j}\right)=\bar{v}_{j}\left(p_{j}\right), j=1,2$. It is easy to see that there exists an equivariant homeomorphism $h: C l\left(W^{u}\left(\sigma_{1}\right) \cap W^{s}\left(\sigma_{2}\right)\right) \rightarrow \cdot C l\left(W^{u}\left(\sigma_{1}\right) \cap W^{s}\left(\sigma_{2}\right)\right)$ conjugating $f$ and $\bar{f}$ with $h\left(x_{0}\right)=\bar{x}_{0}$ if and only if $v_{1}=\bar{v}_{1}, v_{2}=\bar{v}_{2}, g_{j}=\bar{g}_{j}$ for $j=1, \ldots, k$. From this the theorem follows easily.

REMARK. (1) From the proof of the above theorem it follows that the modulus of stability of $f \in \mathscr{B}$ is given by the number of one-dimensional critical elements $\sigma$, with $\operatorname{dim} N\left(G_{x}\right) / G_{x}=1$ for $x \in \sigma$ and the number of $(\mathbf{Z} \times G)$-orbits of nontransversal intersection of strong stable and strong unstable foliations of these critical elements.

(2) The arguments in the above proof can be used to prove a stronger result: $f \in A$ has finite modulus if and only if the strong stable and strong unstable foliations of critical elements are transversal to each other except possibly at a finite number of $(\mathbf{Z} \times G)$-orbits where the tangencies are of finite order.

If $d=2$, then the action of $G$ is transitive and an element $f$ of $\operatorname{Diff}_{G}(M)$ is determined by its value in one point: two equivariant diffeomorphisms are conjugate iff they are equal. Hence if $\operatorname{dim} N\left(G_{x}\right) / G_{x}=0$, then every diffeomorphism is structurally stable; if $\operatorname{dim} N\left(G_{x}\right) / G_{x}=j$, then every diffeomorphism has modulus $j$.

\section{REFERENCES}

[B] E. Bierstone, General position of equivariant maps, Trans. Amer. Math. Soc. 234 (1977), 447-466.

[Br] G. E. Bredon, Introduction to compact transformation groups, Academic Press, New York, 1972.

[dM] W. de Melo, Moduli of stability of two-dimensional diffeomorphisms, Topology 19 (1980), 9-21.

[dM-D] W. de Melo and F. Dumortier, A type of moduli for saddle connections of planar diffeomorphisms, preprint, IMPA. 
[dM-vS] W. de Melo and S. van Strien, Characterizing diffeomorphisms with finite modality on tw'o-dimensional manifolds (to appear).

[dR] G. L. dos Reis, Structural stability of equivariant vector fields on two-manifolds, Trans. Amer. Math. Soc. 283 (1984), 633-643.

[F1] M. J. Field, Transversality in G-manifolds, Trans. Amer. Math. Soc. 231 (1977), 429-450.

[F2] __ Equivariant dynamical systems, Trans. Amer. Math. Soc. 259 (1980), 185-205.

[H] P. Hartman, Ordinary differential equations, Wiley, New York, 1964.

[Ha] _ On local homeomorphisms of Euclidean spaces, Bol. Soc. Mat. Mexicana (2) 5 (1960), 220-241.

[P] J. Palis, A differentiable invariant of topological conjugacies and moduli of stability, Asterisque 51 (1978), 335-346.

[P2] __. On Morse-Smale dynamical systems, Topology 8 (1969), 385-404.

[P-S] J. Palis and S. Smale, Structural stability theorems, Global Analysis, Proc. Sympos. Pure Math., Vol. 14, Amer. Math. Soc., Providence, R. I., 1970.

[P-dM] J. Palis and W. de Melo, Geometric theory of dynamical systems, Springer-Verlag, Berlin and New York, 1982.

Instituto de Matemática Pura e Aplicada, Estrada Dona Castorina, 110 Rio de Janeiro RJ, BRAZIL

Departamento de Matemática, Universidade Federal de Goias, 74000 Goiania Go, Brazil

Departamento de Matemática, ICEx - UfMG, Cidade Universitária, Pampulha 30.000, Belo Horizonte M.G., BraZIL 